Voix et Images

volxetimages

\title{
Espace et délire dans Kamouraska d'Anne Hébert
}

\section{Gabriel-Pierre Ouellette}

Volume 1, numéro 2, décembre 1975

\section{Raoul Duguay}

URI : https://id.erudit.org/iderudit/290078ar

DOI : https://doi.org/10.7202/290078ar

Aller au sommaire du numéro

Éditeur(s)

Les Presses de l'Université du Québec

ISSN

0318-9201 (imprimé)

1705-933X (numérique)

Découvrir la revue

Citer cet article

Ouellette, G.-P. (1975). Espace et délire dans Kamouraska d'Anne Hébert. Voix et Images, 1(2), 241-264. https://doi.org/10.7202/290078ar d'utilisation que vous pouvez consulter en ligne.

https://apropos.erudit.org/fr/usagers/politique-dutilisation/ 


\section{Espace et délire dans Kamouraska d'Anne Hébert}

Élisabeth d'Aulnières, du début à la fin du roman Kamouraska, se trouve dans la maison de son second mari, le notaire Jérôme Rolland, rue du Parloir, à Québec, et la plupart du temps elle est étendue dans la chambre de Léontine Mélançon, l'institutrice des enfants. C'est dans cette pièce que se tient la presque totalité du «discours» de Kamouraska.

Les lieux de l' «histoire" du roman, qui se passe surtout ailleurs que dans la maison de la rue du Parloir, se greffent et se fusionnent souvent à la chambre de Léontine Mélançon, qui devient ainsi le lieu central du texte. Mais, cette chambre disparaît à plusieurs occasions du "discours" et de l'«histoire» de Kamouraska. D'autres lieux y surgissent, s'y installent et la rejettent dans l'oubli pur et simple. Ces lieux se désintègrent par la suite, pour réapparaitre quelquefois et encore s'évanouir.

Les distances sont aussi, par le fait même, abolies; tour à tour, Élisabeth d'Aulnières se revoit et se retrouve soit à Sorel, dans la maison de son enfance, rue Augusta, ou dans la maison du docteur George Nelson ou chez le Gouverneur, soit à Saint-Ours, ou encore au manoir de Kamouraska.

La fusion des lieux fait d'abord revivre un ailleurs désiré, avant que leur destruction fasse sourdre un autre ailleurs, redouté, celui-là, repoussé, mais, enfin, accepté. Cet ailleurs, "de l'autre côté du monde», se trouve aux limites d'un espace sans lieux ou d'un lieu vide qui est le théâtre d'événements vécus, dans le discours du roman, par Élisabeth d'Aulnières qui pourtant ne les a jamais vécus, dans l'histoire du roman.

Élisabeth d'Aulnières revit le passé, d'abord de façon progressive. Elle le refuse même, au début des heures qu'elle passe dans cette pièce «petite et ridicule» $(40)^{1}$ : «Boucher toutes les issues. Demeurer seule avec

1. Les chiffres entre parenthèses renvoient aux pages de l'édition originale de 1970 et à celles de l'édition de 1973 qui est la reproduction photographique de la première édition. Anne Hébert, Kamouraska, Paris, Seuil, 1970, 250 pages. 
mon mal de tête, en guise d'unique compagnie. N'admettre aucune intrusion, aucune autre torture que la migraine. Que personne ni rien au monde n'entre ici. Me reposer (40). Mais elle sait très bien ce qui va se passer, car ce n'est pas la première fois qu'elle se joue ce spectacle ${ }^{2}$, comme elle le dit plus loin: "La même inflexible convocation, hors du temps. Se répétant à intervalles plus ou moins réguliers" (230). La conscience aura donc une certaine prise sur le songe, au début: "Organiser le songe. Conserver un certain équilibre» (97): «Désarmer le génie malfaisant des sons et des images, lui consentir quelques concessions minimes. Tricher avec lui. Choisir mes propres divagations ${ }^{3}$ " (40). Après un certain temps, cette lucidité ne peut plus rien. Elle en arrive à faire des concessions, à se donner comme règle de «respecter l'ordre chronologique. Ne pas tenter de parcourir toute sa vie d'un coup ${ }^{4}$ " (97) et finalement le vocabulaire de la prudence et du calcul cède le pas à celui de l'évidence inéluctable, une évidence qui s'explique par son désir de retrouver la vie heureuse qui a précédé le meurtre de son époux par George Nelson; ce désir l'emporte sur sa crainte de revivre le crime:

L'espace d'une seconde. II suffit d'une seconde. Et ma vie chassée me rejoint au galop. Rattrape le temps perdu, d'un seul coup. Les défenses en moi s'abattent comme des châteaux de cartes (112);

Trop tard. Il est trop tard. Le temps retrouvé s'ouvre les veines (115); Je dis «je" et je suis une autre (115).

Ce temps retrouvé et cette nouvelle identité prennent racine dans un “autre monde» où «une autre vie existe, mouvante et bouleversante » (142). Elle s'est laissée aller au "génie malfaisant des sons et des images", parce qu'elle sait, en somme, qu'elle peut, par lui, retrouver au moins pour un temps la vie elle-même: “J'ai l'air d'évoquer des esprits et pourtant c'est la vie même que je cherche..." (127) Cette vie, qui est ailleurs, elle la retrouve et la possède. Elle précise qu'il n'y a aucun subterfuge de la mémoire: «J'habite ailleurs. Un lieu précis. Un temps révolu. Aucun prestige de la mémoire ne pourrait réussir cela. Il s'agit de la possession de ma vie réelle. De ma fuite parfaite de la rue du Parloir" (163).

Ces phénomènes de fusion des lieux, ce phénomène d'ubiquité, sont perçus graduellement par Élisabeth d'Aulnières. Des bruits, venant de

2. II s'agit bien de spectacle: la salle d'audience, lors du procès, est un «cirque" (48) ; la maison de Sorel est "pareille à un tréteau» (54); son mariage avec Antoine Tassy a des allures d'un théâtre de marionnettes: "Voici la mariée qui bouge, poupée mécanique... Le marié est en bois colorié. La mariée aussi, peinte en bleu» (71); elle dit d'Aurélie Caron et d'elle-même: "Nous avons l'air de répéter une pièce" (61); plus loin, elle dira: "Comme si je n'attendais plus que ce signal, j'entre en scène» (115).

3. C'est moi qui souligne. Il en sera ainsi pour tous les mots soulignés.

4. Même alors, elle sait que toutes ces précautions sont inutiles, car elle dit, dans la même page: "Surtout ne pas passer en jugement! Pas tout de suite! Repousser l'échéance. » 
l'extérieur ou de l'intérieur de la maison, déclenchent d'abord chez elle de simples réminiscences et, en même temps, cette crainte de «se laisser emporter par un rêve", de "rêver au risque de se détruire, à tout instant, comme si on mimait sa mort" (23). Le bruit d'une charrette dans la nuit lui rappelle le passage du docteur Nelson sous ses fenêtres ${ }^{5}$ mais en plus de cette charrette, il y a le coup de la sonnette de la porte d'entrée qui répond au coup de sonnette pour appeler Florida, la bonne ${ }^{6}$, il y a ses doutes sur la réalité de tous ces bruits, il y a la présence vérifiée de la charrette devant la porte d'entrée ${ }^{7}$ et cela fait entrer des façons du songe dans la réalité, avant que la réalité s'incorpore au songe. Le bruit que fait Florida en déplaçant des meubles ou en ouvrant la porte cochère, fait sourdre inopinément ce songe redouté: "Le songe! Est-ce le songe?" (32) et, songe ou non, FLorida devient le huissier de la cour qui lit en anglais l'acte d'accusation. La voix de sa petite fille, Anne-Marie, "tire au jour une autre voix enfouie dans la nuit des temps. Une longue racine sonore s'arrache et vient avec la terre de [sa] mémoire. L'accent rude et effrayé de Justine Latour qui témoigne devant le juge de paix» (34-35): les prestiges de la mémoire ont un rôle important, ici, mais Élisabeth d'Aulnières précise que si cette racine sonore vient avec de la terre de la mémoire, elle s'est par ailleurs arrachée du sol de la mémoire, comme pour lui accorder l'autonomie nécessaire pour devenir un des éléments qui formeront le temps retrouvé.

D'autres sons peuvent faire partie intégrante du temps retrouvé ou bien venir relancer cette fusion des lieux du passé au lieu présent. Ainsi, quand elle dit: «On marche dans le corridor, un petit pas décidé. C'est Aurélie qui..." (43), il peut s'agir du corridor de la maison de Sorel et de celui qui passe devant la chambre de Léontine Mélançon; la même ambiguïté se retrouve, quand elle note: «Immédiatement le rire d'Aurélie me glace. Cela vient de quelque part dans la maison. Perce les murs. On doit l'entendre du dehors" (107); s'agit-il du rire d'Aurélie qu'elle imagine dans la maison du notaire Rolland, ou d'un souvenir du rire d'Aurélie dans son ancienne chambre de la rue Augusta, à Sorel, où elle se retrouve dans son songe? Dans la scène du tribunal transformé en arène de cirque, les roulements de tambour, les chapelets qui "sonnent comme des grelots" et $l$ ' «orage de rire"sont du domaine du rêve ${ }^{8}$, mais quand $\mathrm{Mme}$ Rolland se voit, aussitôt après, la cible de poignards, quand elle «cherche éperdument l'issue cachée pour sortir de ce cirque" et quand, croyant "enfin se réveiller», elle "porte la main à son sein» et "éprouve une vive douleur», le rêve débouche sur une sensation consciente et ne laisse pas, même après des manifestations extrêmes, d'être lié au réel, comme pour préparer à une réalité exacerbée, une réalité de "rêve», celle du meurtre qui sera vécue dans la confusion du temps et de l'espace.

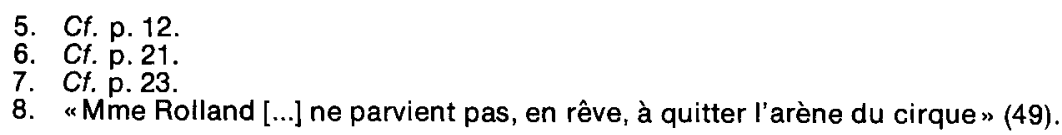


Cette interaction du réel et de l'imaginaire trouve un pendant formel dans l'écriture d'un autre texte où des éléments sonores interviennent; elle compare le bruit d'une porte à un bruit dans un lieu désert et c'est la comparaison qui devient l'élément principal et éclipse la chose comparée (le bruit): "La porte d'entrée claque ${ }^{9}$. L'écho derrière la porte fermée est extraordinaire. Le bruit de la porte claquée persiste longtemps, ainsi que dans un lieu désert, sans meubles ni rideaux. Un lieu immense. Démesuré. Une espèce de gare. Une cave haute et nue. Presque aussitôt une voix pointue s'élève, reprise à l'infini par l'écho " (56); les pistes se brouillent, l'écho d'un bruit et la notation de ce qu'il évoque, transportant Élizabeth d'Aulnières d'un lieu dans un autre.

Le phénomène de l'écho encadre cette transformation de l'espace, comme jusqu'ici les bruits et les sons ne faisaient que suggérer ou provoquer, en les encadrant en quelque sorte, des réminiscences de lieux et de situations. Les odeurs mettent davantage en évidence la réalité des sensations d'Élisabeth d'Aulnières et sa perception sensorielle de ce monde révolu.

Elle évoque les parfums de la nature, comme elle évoque ceux des chambres fermées ${ }^{10}$. Ces odeurs sont reliées à des lieux précis. Elles sont des présences actives ${ }^{11}$, s'élèvent, emplissent l'air environnant; elles la font suffoquer. Si cette odeur lourde des roses n'est que le rappel d'un malaise passé, quand elle dit, au sujet du cabinet de toilette de sa mère: «On étouffe ici. Cette odeur de renfermé. J'ai la nausée " (133), le temps présent des verbes indique un malaise actuel de Mme Rolland et non un malaise passé de la jeune Élisabeth. Ces odeurs se retrouvent en elle, autour d'elle, et lui font retrouver sa sensibilité d'antan: "Je redeviens sensible à outrance" (71). Elle dira plus loin, en parlant de la maison de la rue Augusta, à Sorel: «Je respire un air raréfié, déjà respiré » (100); il ne s'agit plus de la répétition d'une sensation passée, mais d'une sensation présente qui dépasse les seuls pouvoirs de la mémoire et si cet air raréfié est celui de la chambre de Québec, il n'en reste pas moins qu'elle voit un lien nécessaire entre cette odeur et la chambre de Sorel.

$\mathrm{Ce}$ sont encore des sons et des odeurs qui entremêlent la pluie sur Québec et une autre pluie qui tombait à Sorel. Ces «grandes gifles de pluie" qui "s'écrasent sur la vitre», cette rue «pleine de flaques», ce peut être à Québec comme à Sorel ${ }^{12}$; aussitôt après quand «l'odeur de la pluie se mêle à la senteur aigre de l'encre, au goût fade de la feuille de papier

9. S'agit-il, encore ici, de la maison de Québec ou de celle de Sorel ?

10. "L'odeur du foin coupé. Le parfum des trèfles" (71); «Le bas du fleuve respiré à plein poumons. [...] L'odeur des grèves se lève, de plus en plus fort " (74); "L'odeur de la marée basse emplit l'air lourd" (75); "Une odeur suffocante de roses fanées. Un relent de souris empoisonnées, sous la plinthe du vestibule" (97); "ll y a une odeur d'huile et de fumée qui emplit la cuisine du docteur» (153).

11. Anne Hébert, les Chambres de bois, Paris, Seuil, 1958, p. 94: "ll y eut une minute de silence au cours de laquelle l'odeur de l'appartement s'empara de tout l'espace, comme une bête familière qui n'en finit pas de manifester bruyamment sa présence.»

12. Pour tout ce passage sur Sorel et Québec sous la pluie, cf. p. 152-153. 
blanc... ", on est à Sorel, avec Aurélie Caron, et Élisabeth d'Aulnières se dit "prisonnière sous la pluie"; "le bruit des gouttes d'eau sur le toit de bardeaux" évoque sûrement, par le contexte, le bruit de la pluie sur la maison du docteur Nelson, bien qu'il peut être confondu avec celui de la pluie à Québec; le «fracas» de la gouttière qu' «il faudrait faire réparer", enpêche d' ' habiter la campagne mouillée de Sorel » et ramène à Florida et au notaire Rolland qui se meurt, rue du Parloir. On connaît déjà ces allers et ces retours des lieux du passé aux lieux du présent, mais la pluie semble contenir tous ces lieux sous un seul espace; “Si loin que je sois dans l'espace et le temps, je demeure attachée à George Nelson, en cet instant précis où toute la campagne de Sorel chavire sous la pluie. " II est à noter que son éloignement dans le temps devient, sans transition, «... cet instant précis où toute la campagne de Sorel chavire sous la pluie». Si l'esprit confond deux lieux sous la pluie, leurs habitants n'en passent pas encore les limites: “Si je retrouve son visage c'est à travers une vitre. Une eau profonde, infranchissable."

Cette sensation de se retrouver dans les lieux du passé ou de se trouver dans un état de «mouvance» entre le présent et le passé, est renforcée par des comparaisons avec le mouvement sur l'eau ou dans l'eau. Elle est sur un «chemin de halage» avec Aurélie Caron; ce chemin est évidemment le long d'une rivière. Elle est plongée tout à coup dans une pénombre. Cette pénombre humide et le mouvement du courant créent en elle des sensations qui se confondent et de là, naît l'impression d'être emportée par la rivière:

Je passe brusquement du soleil aveuglant à une sorte de pénombre, humide, envahissante. Une seule idée en vrille dans ma tête: il faut que je rentre [...] je m'éloigne vertigineusement d'Aurélie. Sans parvenir à faire un pas de moi-même d'ailleurs. C'est comme si je filais sur la rivière. Une sorte de radeau plat sous mes pieds. La rivière silencieuse. Aucune résistance de l'eau. Aucun bruit de vagues ou de rames. Je vais au bal du Gouverneur. II faut que j'aille au bal du Gouverneur (63-64).

La puissance de la mémoire, ou encore du passé, est remplacée ici par la puissance de l'eau.

Cette eau qui transporte, dans le passé, d'un lieu à un autre, ne fait pas oublier l'état de rêve; elle est «parée comme une noyée que l'on va balancer par-dessus bord. Immergée dans le rêve saumâtre" (80). L'eau montre aussi qu'elle résiste au songe, comme le songe lui résiste: «Un pas devant l'autre, avec peine. Comme si j'avançais dans une eau épaisse, étrangement résistante" (96); elle ose franchir, alors, la clôture invisible qu'elle sent dressée entre elle et sa chambre, rue Augusta, à Sorel, et elle le fait sur l'invitation insistante d'Aurélie. Dans le songe, celle-ci devient une sorte de meneuse de jeu qui, avant que tout ait lieu, sait déjà tout ${ }^{13}$. Elle est la «mauvaise conscience" d'Élisabeth d'Aulnières: c'est elle qui dit la vérité au procès et elle n'est pas dupe des airs de «saintes nitouches » (96) de sa

13. "Elle sait la suite de l'histoire et jure que mes intentions ne sont pas pures " (96). 
maîtresse; elle apparaît dans le songe pour que les choses soient telles qu'elles étaient. La mémoire d'Élizabeth d'Aulnières est "contrôlée" par le songe ${ }^{14}$ et, ainsi, le songe et la mémoire commencent à se nuire l'un l'autre.

Une eau infranchissable la séparait de George Nelson; il n'y a pas ces barrières ou ces espaces interdits entre elle et Aurélie, ses vieilles tantes ou sa mère, car elles, elles ne sont pas de cet «autre côté du monde", dans cet espace inconnu où le songe la mènera, malgré toute sa résistance. Pour le retour aux lieux qu'elle a occupés avec son époux, le songe-souvenir renferme autant d'ambiguïté que pour leurs rapports affectifs. L'Antoine Tassy du songe, elle le craint: elle craint sa vengeance, qu'il venge sa mort sur elle, la complice de George Nelson; l'Antoine Tassy du souvenir, elle le désire au point qu'elle préfère "affronter son affreuse colère de bête abattue. La vengeance d'Antoine. Tout plutôt que de retrouver ce clair regard d'enfant, parfois si bleu. Cette espèce d'étonnement triste ${ }^{15}$ " (85). Ainsi, de la chambre conjugale, au manoir de Kamouraska, elle revoit surtout «un fragment de miroir ${ }^{16}$ » et ce fragment est à la fois limite et ouverture, comme les relations des époux sont à la fois désir et haine. Tout autour de ce morceau de glace, règne la violence. Antoine «brandit le poing audessus de [la] tête [d'Élisabeth]. Pour [la] maudire» (85); «Le morceau de glace se casse en mille miettes" (85); "Il [lui] lance un couteau de cuisine par la tête» (86); il «tire une balle dans la glace. La rate» (87). Le fragment lui-même est "un petit hublot de tain pur ${ }^{17}$ ", mais on ne voit pas à travers ce hublot recouvert de tain: c'est une "eau morte» où se mirent le père et la mère avec leur nouveau-né. Élisabeth d'Aulnières semble, avec ce fragment, trouver un point d'appui pour la poursuite du songe à travers le souvenir: un élément de l'espace du songe garde des images de passé ${ }^{18}$ et le passé, de cette façon, se nourrit lui-même. Mais ce point d'appui est très fragile et très limité: ce «hublot" est une fausse fenêtre et comme le dit la belle-mère, Mme Tassy: «-Tout ça, c'est du théâtre!» (85), du théâtre de "marionnettes". L'espace du songe risque de se refermer.

Cependant, la glace se brise, sans intervention aucune: «Un seul éclat persiste au mur." La clarté, la limpidité (une qualité de l'eau), le trop de pureté, l'éclat perçant de cette glace font fermer les yeux à Élisabeth, parce qu'elle veut «tout plutôt que de retrouver ce clair regard d'enfant, parfois si bleu». Le miroir est devenu le regard bleu d'Antoine (avant, elle disait de la glace: "Son éclat ne peut que me percer le cœur»). C'est devant ce mor-

14. C'est quelques lignes plus loin qu'elle veut "organiser le songe» (97); un peu avant, elle se jugeait "toute intoxiquee de songe" (95).

15. C'est dans les lignes suivantes qu'elle revit leurs étreintes sur la grève, comme "deux enfants sauvages" (86).

16. L'œil va maintenant jouer le rôle important.

17. Ce hublot continue la métaphore du vaisseau appliquée au manoir: "Le manoir est illuminé dans la nuit. Comme un vaisseau retiré de la mer. Hissé sur un cap. En radoub. Conservant toutes ses lumières" (84).

18. Cf. Anne Hébert, Poèmes, Paris, Seuil, 1960, p. 89: "Longtemps nous avons gardé des jours anciens en liberté dans les chambres du fond / Les avons lâchés dans toute la maison, livrés au temps et remis en marche comme des songes / Se sont promenés de chambre en chambre, toute figure reprise à mesure au fil des miroirs $/$... 
ceau pur comme son regard qu'Antoine tient à répéter sa confession, un peu plus loin: Antoine face au regard pur d'Antoine. Les objets de la mémoire ont une vie propre, dans le songe. lls ne sont pas tels que dans le passé; ils semblent avoir vécu eux aussi à travers le temps et être parvenus jusqu'au temps présent.

Il y a un miroir dans un autre lieu du passé des deux époux, le cabinet de toilette de la mère d'Élisabeth d'Aulnières. Il n'est pas une «eau morte "; il est «ravivé comme une source» (134). Cette fois, un élément du passé a subsisté tel quel, bien qu'il faut nettoyer la glace, recouverte de poussière ${ }^{19}$. Elle a gardé le passé intact: «Ma jeunesse sans un pli » (134). Elle est un élément de la scène qu'Élisabeth d'Aulnières revoit: toute cette scène s'est réellement passée face à cette glace. Elle occupe tout l'espace de la mémoire, comme elle encadrait toute la scène au moment où elle a eu lieu. Cette fois, l'objet du passé ajoute à sa qualité de réfléchir un tableau, celle de faire revivre toute une situation passée ${ }^{20}$. Et ce miroir fait bien partie du processus du songe-souvenir, parce qu'Élisabeth d'Aulnières continue à faire ses réflexions face à ce qu'elle revoit: "Un homme et une femme côte à côte. Mari et femme. Se haïssent. Se provoquent mutuellement. Dans une lueur douce de bougies, allumées de chaque côté du miroir» (134). Finalement, "la glace se brouille" (135). Le miroir, eau morte ou source, fait revivre momentanément le passé, comme le font une photographie ou un film, mais il n'a pas cette faculté de faire renaître dans l'espace environnant les choses et les personnes du passé, comme le peut la lumière. D'ailleurs, si la glace se brouille, la raison en est, sans doute, que "quelqu'un souffle les bougies» (135), car, d'abord et avant tout, la mémoire est une «lanterne sourde, brandie à bout de bras » (142); s'il n'y a pas de lumière, il ne peut y avoir de mémoire, ni de songe.

Avant que la lumière ne jette ses rayons sur les choses et les fasse apparaître, elle environne Élisabeth d'Aulnières, l'aveugle et la blesse. D'abord, le soleil fait une «lueur étrange, couleur jus de framboise» (40) à travers les rideaux de toile rouge, dans la chambre de Léontine Mélançon. Cette lueur - et cela demeure inexpliqué - augmente, devient « une sorte d'éclat qui monte peu à peu et s'intensifie à mesure» (50), un “éblouissement» (50), un «blanc aveuglant. Toujours ce soleil qui...» (80).

Cette lumière se transforme de «lueur étrange», en «images monstrueuses" (41). En blessant les yeux d'Élisabeth d'Aulnières, elle lui brouille la vue et ses «rayons bardés de feu», ses «rayons pointus qui déchirent [ses] yeux", amènent avec eux "des femmes minuscules en tabliers et bonnets blancs», "des espèces de petits êtres»; il s'agit des trois domestiques, Justine Latour, Sophie Langlade et Aurélie Caron. Élisabeth d'Aulnières le dit bien:

19. Cf. p. 133.

20. "Comme si je voyais un grand miroir aux images ineffaçables retenir mes gestes et mes regards. " Cf. Anne Hébert, le Torrent, Montréal, Beauchemin, 1950 , p. 51 . (Cité dans Guy Robert, la Poétique du songe, Montréal, Cahiers de l'ÁGEUM, 1962, $n^{\mathrm{c}} 4$, p. 54. 
Cest à cause de la lumière. C'est un phénomène de la lumière. Cela est entré dans la pièce avec ces rayons pointus qui déchirent mes yeux. Ces images monstrueuses, aiguës comme des aiguilles. C'est dans ma tête qu'elles veulent s'installer. Me tourner de côté, ouvrir les yeux. Ne pas leur permettre de prendre racine, les arracher de mes yeux, ainsi qu'on extirpe une poussière. Je n'arrive plus à bouger. Mes paupières sont lourdes. Semblables à du plomb. Ce doit être la poudre du docteur. Encore un petit effort. Je parviendrai bien tout de même à me tourner de l'autre côté. Voilà. Enfin. Ça n'a pas été sans peine. C'est bien ce que je craignais, les trois femmes ont grandi. Grandeur nature elles envahissent à présent la petite chambre de Léontine Mélançon. Je crois qu'elles font le ménage et disposent des objets sur la commode, comme des pièces à conviction ${ }^{21}$.

L'entrée de ces personnages, causée par la lumière, commande le retour de tous les lieux du passé. On y reviendra.

Cette lumière ne se transforme pas seulement en «images vivantes", mais, en augmentant d'intensité, elle devient la lumière ou même le soleil du songe, au-dessus des lieux, des maisons, des personnages qui prennent alors plus d'autonomie.

Cela peut commencer par la sensation qu'on a, en levant la tête vers le soleil, les yeux fermés: "La lumière me blesse toujours. Je la sens en aiguilles rouges, brûlantes, sous mes paupières fermées. Comme lorsqu'on lève la tête vers le soleil en plein midi» (109). Cette brûlure et cette couleur se transforment en une sorte de décor de théâtre: «Il y a un soleil qui bouge au ciel. Une lueur rouge plutôt qui fait semblant d'être le soleil. Simule le déroulement régulier des jours et des nuits" (142). Ce décor, à son tour, devient un mécanisme qui fait surgir des ténèbres la maison de la rue Augusta, à Sorel:

Depuis un instant, il y a quelque chose qui se passe du côté de la lumière. Une sorte d'éclat qui monte peu à peu et s'intensifie à mesure. Cela devient trop fort, presque brutal. J'ai envie de mettre mon bras replié sur mes yeux, pour les protéger contre l'éblouissement.

Soudain, cela s'arrête et se fixe sur une seule maison de briques rouges, angle des rues Philippe et Augusta. Isolée de ses voisines, par cette clarté qui lui tombe dessus, la maison se met à briller. Précise comme si on la regardait à la loupe. Lustrée. Émaillée de lumière. Le petit jardin derrière pâlit sous un si grand soleil. Les hortensias bleus paraissent poudrés de blanc. Deux étages de briques. Des volets de bois verts, strictement fermés. Une galerie de bois, les minces colonnes. Le fronton légèrement découpé dans le bois, fine dentelure peinte à la chaux, si blanche, si fine et folle. Je pourrais y toucher. Chaque dent, chaque nervure, vivante dans un éclairage terrible et fort, dur, vif, jaune. Un soleil fixe au-dessus de la maison, un peu à gauche (50-51).

21. «Une seule maison demeure tout illuminée. Pareille à un tréteau. La moindre poussière vole avec la précision d'une phalène, autour d'une lampe. L'air, luí, ressemble à la lumière, clair, et sonore» (54); "Sorel à nouveau. La maison de la rue Augusta, ouverte au beau soleil d'octobre. On m'attend. La lueur des feuilles rousses emplit la rue bordée d'arbres. Un courant d'air léger, coloré, circule avec la lumiere» (95). 
Ce soleil fixe montre la grande part que le théâtre tient dans ce songesouvenir, mais il apparaît aussi de façon évidente, dans cet extrait, que la lumière - et seule la lumière - fait voir un espace en son entier, avec tous ses détails. C'est «du côté de la lumière», de la lumière vue comme un espace, que tout commence et c'est d'elle qu'émane cette «sorte d'éclat» qui isole la maison de la rue Augusta, dans un «centre lumineux" (51) et lui donne les qualités de la lumière. Élisabeth d'Aulnières dit plus loin: "ll n'y a que ma maison de la rue Augusta, au coin de la rue Philippe, qui émerge étincelante, comme un éclat de verre" (51). Le tableau de cette maison plongée dans la lumière revient au moins deux autres fois, dans le texte du roman ${ }^{21}$.

A l'intérieur de la maison, la lumière est aussi présente: «extraordinaire [...], intolérable, plus claire que le soleil» (103); «...de plus en plus violente, [elle] tombe maintenant en faisceaux du plafond" (107); «ll y a trop de soleil dans cette maison aussi. Antoine ne tente aucun geste pour se retirer de la fenêtre où il se tient debout, exposé en pleine lumière. Ses yeux rougis ne clignent pas. On dirait qu'il se soumet lui-même, sans aucune espèce de défense, au supplice de la lumière. / Un long rayon traverse la pièce, m'atteint de plein fouet. Je suis prise au piège de la lumière à mon tour" (146-147).

La lumière piège les personnages, comme elle "se fixe" sur la maison de Sorel, et elle est la cause première et la condition de ces retours dans le passé, comme Aurélie le dit: «- On ne peut pas toujours vivre dans la noirceur. II faut ce qu'il faut. Les grandes scènes de votre vie s'en viennent Madame. C'est en pleine clarté qu'il faut les revivre» (103). A un autre moment, Élisabeth d'Aulnières, lors d'une de ses nombreuses réticences à accepter ces retours en arrière, décrit ce qui se passe à ces moments et le mot «cendres» la conduit à voir la vie retrouvée comme une substance en flammes et, par conséquent, comme une substance lumineuse, ce qui montre encore l'importance, du moins visuelle, de la lumière: «Je voudrais fuir. Ne pas rentrer à l'intérieur de la maison. Risquer à coup sûr d'y retrouver ma vie ancienne se ranimant, secouant ses cendres en miettes poudreuses. Chaque tison éteint, rallumé. Chaque rose de feu éclatante, foudroyante » (56).

Évidemment, la présence de la lumière n'est pas toujours signalée dans le songe-souvenir, mais c'est elle, les sons, les odeurs et les propriétés de l'eau et du miroir qui rendent possible, pour Élisabeth d'Aulnières, la reconstitution sonore, olfactive, tactile et visuelle du passé. Une fois que le mouvement a été donné, tous les lieux du passé se reconstruisent devant elle ou en elle. Ce n'est pas encore un voyage dans le temps; ce qu'elle voit, là où elle se voit, ce ne sont pas les lieux de son passé tels qu'ils étaient, mais leur reconstruction à partir des cendres du passé ou leur restitution sur le tain des glaces.

Des lecteurs qui préféreraient insister sur l'aspect imaginaire du songe plus que sur les «réalités " de cet imaginaire, ne peuvent cependant pas nier qu'Élisabeth d'Aulnières voit et ressent ce qui se passe autour d'elle, en toute conscience et en toute lucidité. Elle y est forcée, comme elle 
le dit, mais avec l'attention de tout son être et cette attention se porte, à ce moment, sur la présence, au lieu et à la place de la chambre de Québec, de celle de Sorel:

Ce n'est pas tant la netteté des choses en soi qui me bouleverse. Mais je suis forcée (dans tout mon être) à l'attention la plus stricte. Rien ne doit plus m'échapper. La vraie vie qui est sous le passé. Des fines piqûres d'insectes apparaissent dans le bois du lit, vermoulu. La chambre tout entière est rongée. Elle tient debout par miracle et s'est déjà écroulée. A été remise sur pied, exprès pour cet instant aveuglant. Tant de précision...22 (104)

Ces phénomènes se passent bien en elle et autour d'elle, dans son visage et dans un espace qui peut être en même temps proche et très lointain: "Antoine soudain est debout en face de moi qui me regarde. Cette stupeur sans borne. Son dernier regard. Trop de lumière. J'ai la pudeur de ma haine et je baisse les yeux à nouveau. II parle tout bas, à travers ma face aveugle. Sa voix éteinte lente et pourtant menaçante semble venir d'un point éloigné dans l'espace" (147).

Elle perçoit, en plus des différences de perspectives, des transformations dans les lieux où elle se retrouve: "ll me semble que mon lit ${ }^{23}$ est plus haut que d'habitude. On dirait qu'il se trouve placé sur une sorte d'estrade. La lumière, de plus en plus violente, tombe maintenant en faisceaux du plafond, au-dessus de mon lit. Je pourrais me croire sur une table d'opération" (107). On a la chambre, la hauteur du lit, l'estrade et, finalement, la table d'opération. Les espaces du songe ont une vie propre: la vie du passé, bien sûr, comme elle le dit: "La vraie vie qui est sous le passé ", mais aussi une force vitale qui leur vient du songe même et de celle qui songe, une force qui défiera bientôt les lois du temps et de l'espace. Ces lieux, ces différentes perceptions d'un même lieu, proviennent d'un seul et même espace: la chambre de Léontine Mélançon, à Québec.

Après qu'Élisabeth d'Aulnières, par l'effet des rayons du soleil à travers ses paupières, a vu les trois domestiques envahir la chambre, elle note ceci : «C'est très étrange. Les objets de Léontine eux-mêmes changent doucement. Je suis sûre que ce ne sont plus tout à fait les mêmes. Imperceptiblement, ils deviennent autres " (41). Après l'intrusion des personnages, ce sont les objets qui se transforment. Par la suite, les domestiques «transportent des meubles sans effort, [...] Elles n'ont ni poids ni consistance. Je suis sûre qu'on pourrait passer le bras au travers de leurs corps, comme on transperce un nuage. Voici qu'elles déplacent un piano droit, le roulent jusqu'à mes pieds " (42). Les lieux du passé occupent peu à peu la chambre où se repose Élisabeth d'Aulnières. Plus loin, les trois domestiques «ouvrent des portes à l'infini, se croient obligées de faire communiquer entre elles toutes les pièces de la maison. Mystère des chambres en enfilade. Elles [lui] font signe et [la] prient, d'un air malicieux, d'habiter bien

22. Il faut rapprocher ce texte de celui de la page 51, cité plus haut, et noter dans ce dernier la phrase: "Je pourrais y toucher " et la qualité de "vivante " donnée à chaque dent et à chaque nervure du fronton de la maison.

23. Dans le songe, elle est dans une chambre de la rue Augusta, à Sorel. 
vite, à nouveau, toute la maison de Sorel, sans en excepter aucune pièce " (43-44). Le songe-souvenir (ou le somnifère?) peut ainsi tirer du passé un espace d'hier et “occulter » l'espace d'aujourd'hui.

Cette reconstitution des lieux de son enfance, d'abord refusée et finalement acceptée, la mène à ce qu'elle redoute et désire à la fois : revivre ses amours avec le docteur George Nelson. Pour que le passé et le présent de Mme Rolland, Sorel et Québec, ne fassent plus qu'un, il suffit qu'elle revoie la nuque et les yeux noirs du docteur, qu'elle passe ses "deux bras autour du cou de cet inconnu qui sent le tabac frais ou qu'elle respire l'odeur de son gilet ${ }^{24}$ : «Ma folle jeunesse s'ajuste sur mes os. Mes pas dans les siens ${ }^{25}$. Comme on pose ses pieds dans ses propres pistes sur la grève mouillée" (115). Dans le songe-souvenir, le personnage de Mme Rolland disparaît; il n'y a plus que la jeune Élisabeth d'Aulnières-Tassy: «Foulée aux pieds la défroque de Mme Rolland. Aux orties le corset de Mme Rolland. Au musée son masque de plâtre. Je ris et je pleure, sans vergogne. J'ai des bas roses à jours, une large ceinture sous les seins. Je me déchaîne” (115). Elle ajoute ce qui est à la fois une indication de son état passé et présent: «J'habite la fièvre et la démence, comme mon pays natal. J'aime un autre homme que mon mari » (115).

Si elle a connu la fièvre et la démence, en aimant George Nelson, elle les connaît encore en revivant ces amours, car les lieux de cette vraie vie du passé, ces lieux de son enfance et de son amour heureux, patiemment et prudemment reconstruits dans ce songe et ces souvenirs, s'effondrent: «Sorel. La rue Augusta. Ce lieu d'asile est peu sûr. Le refuge de ma jeunesse est ouvert, éventré comme une poupée de son. Toutes les ramifications, les astuces, les tours et les détours de la mémoire n'aboutissent qu'à l'absence" (197). Cette absence est l'absence de son amant, parti tuer son mari, Antoine Tassy, à Kamouraska.

Cette absence va de pair avec une présence qui ne fait qu'amplifier le sentiment de l'absence de George Nelson et la rendre définitive: l'arrivée des témoins des faits qui entourent le meurtre. Ceux-ci semblent profiter de la destruction de la maison de Sorel (ou l'ont-ils causée?) pour s'installer à la porte de la chambre, la seule pièce qui reste debout: "Les témoins attendent impatiemment, derrière la porte de ma chambre. La maison tout entière de la rue Augusta est envahie, dévastée. Détruite de fond en comble. Ma mère et mes tantes étouffent sous les décombres " (207). La présence des témoins est à ce point réelle, dans le songe (fièvre ou démence?), qu'elle ajoute: «Pourvu que les témoins ne se frayent pas un passage rue du Parloir, à Québec, chez mon mari qui est si malade! ”

24. Cf. p. 112.

25. Il faut entendre ici : Mes pas dans les pas de ma folle jeunesse. 
Ce retour à la réalité n'est qu'un détour, une pause, avant de s'enfoncer davantage dans cet espace du meurtre d'où les témoins surgissent. Elle a tout fait au début pour situer le lieu du meurtre dans un "ailleurs" sans contact avec son monde à elle. C'est même l'encouragement qu'elle donne mentalement à George Nelson, quand elle songe à envoyer Aurélie Caron empoisonner Antoine Tassy: «Et puis, tu verras, tout se déroulera dans un autre monde" (173). Quand l'empoisonnement échoue et que Nelson est parti tuer Tassy, elle se rassure, face à l'étrangeté de ce duel à mort commencé il y a longtemps entre les deux hommes, en le rejetant loin d'elle: "Tout cela d'ailleurs se passe hors de moi. Dans un pays lointain, plein de neige et de sang. Entre deux hommes liés entre eux par leur redoutable mystère étranger ${ }^{26} "(202)$.

Cependant, cette notion d'«ailleurs", pour Élisabeth d'Aulnières, a trois sens différents qui montrent la charge d'affectivité que ce mot possède pour elle et l'état d'indécision où elle se trouve. Heureuse d'avoir fui la rue du Parloir et de posséder sa «vie réelle», dans le Sorel d'il y a plus de vingt ans ${ }^{27}$, elle dit habiter "ailleurs. Un lieu précis» (163); quand elle a subitement la nausée dans ces odeurs du passé, elle se rétracte, en pensée, et déclare que «la vraie vie est ailleurs; rue du Parloir, au chevet de [son] mari" (133). Les deux fois, le terme vague d' "ailleurs" est précisé aussitôt qu'il est dit; elle est consciente que sa vie réelle, sa vraie vie, n'est ni à Sorel, ni à Québec. De peur que cette expression de l'«ailleurs " ne mène sa pensée trop loin, elle la corrige et lui donne des limites raisonnables et connues: Québec ou Sorel. Peu importe qu'elle se contredise, pourvu que cet ailleurs ne soit plus là où elle se voit et qu'il soit pourtant là où il est décent de se voir, d'être vue et de mener sa vraie vie: "Marcher, marcher, sans fin. On se retourne sur mon passage. C'est cela ma vraie vie. Sentir le monde se diviser en deux haies pour me voir passer " (8).

Au cours du même songe et des mêmes souvenirs, transparaît une vague conscience d'un ailleurs qui, alors, est bien un «autre» lieu, presque indéfini. Il est autre parce qu'il n'a pas encore de nom ou bien qu'on ne veut ou ne peut pas le nommer, mais c'est un lieu où sa vie s'est comme scellée à jamais: «Ma vraie vie est ailleurs. Toute retirée dans un lieu vague. Une espèce de campagne désaffectée où l'effroi fait des ombres chinoises " (109-110). Ces paroles lui échappent quand elle revit sa première rencontre avec le docteur Nelson, celui qui la «conduira» dans cet ailleurs, celui à qui elle veut dire, plus tard, lors d'un silence qui sépare: "Hors de ce monde, si vous le désirez. C'est là que je vous donne rendez-vous. Telle qu'en moi-même, absolue et libre. Étrangère à tout ce qui n'est pas vous " (123). Sa liberté est ailleurs, dans un lieu autre que tous ceux de ce monde. Pourtant, quand elle connaît, durant le songe, cet ailleurs, hors de ce monde, c'est la maison de Sorel, où elle épie le retour du docteur Nelson, qui redevient pour elle le lieu de sa «vraie vie", sa vie d'amante passion-

26. Déjà, lorsque l'idée du meurtre germe dans leur esprit, elle le voit "dans un champ clos entre Antoine et [George]. Hors de ce monde" (149).

27. Le procès a lieu en 1840 ; elle s'est remariée deux ans aprés; il y a bientôt dix-huit ans qu'elle est mariée à Jérôme Rolland; cf. p. 7, 8, 9 . 
née: «Docteur Nelson, j'épie votre retour. Les prières des agonisants résonnent trop fort, dans mon oreille. Risquent de m'attirer hors de ma vraie vie, de me ramener incessamment dans ma maison de la rue du Parloir " (238). Malgré tout, elle feint “d'appartenir au monde des vivants" (248), car cet ailleurs de liberté et d'absolu est «hors de ce monde».

La destruction des lieux reliés à l'enfance et à l'amour heureux, laisse une place vide où s'engouffre cet ailleurs, situé hors du monde. Elle le désire, elle ressent sa présence, elle le voit et elle y vit.

Cet espace est perçu comme un vide: «J'habite le vide absolu " (197) et ce qu'elle voit de sa fenêtre, rue Augusta, à Sorel ${ }^{28}$, dans le refuge éventré de sa jeunesse, devient pour elle le reflet de ce vide absolu qu'elle ressent à cause de l'absence de George Nelson; le paysage est vide de toute particularité: «Un désert de neige, chaste, asexué comme l'enfer [...] vaste étendue blanche, dépouillée de ses villages et de leurs habitants " (197198); il n'y a plus que «les grandes forêts. Les champs. Le fleuve gelé. [...] La neige étale, à perte de vue ${ }^{29}$, nivelant paysage, ville et village, homme et bête" (198). Il y a similarité entre le vide absolu qu'elle habite et «la neige, à perte de vue, comme un naufrage $"$ (184). La neige nivelle, détruit, tout comme le départ de George Nelson a créé l'absence. Mais, comme elle le dit, le matin du départ d'Aurélie Caron pour Kamouraska: «La neige. Ce n'est pas encore la fin du monde. Ce n'est que la neige" (184), ce n'est pas le vide absolu. Dans le songe, elle habite ce vide pour rejoindre Nelson qui, dans le temps passé, se dirige vers ce vide: «jusqu'au bout du chemin. De tous les chemins. Là où l'horizon bascule sur le vide. Tuer un homme à la limite de ce vide. Se maintenir en équilibre au bord du gouffre» (190).

Cette différence est importante: au moment où Élisabeth d'Aulnières dit habiter le vide absolu, à cause, entre autres, de l'absence prolongée de son amant, le vide n'est qu'une menace pour lui et non une réalité. Elle tente, dans le songe, de procéder d'un vide analogue à celui d'où, lui, il partirait pour revivre l'épisode du meurtre, car, maintenant que, dans la réalité, tout est fini, il est “de l'autre côté du monde ${ }^{30}$ " (189). Pour créer ce vide, elle procède de l'impasse où se trouve le songe, quand tout se détruit; elle procède de son manque total de souvenirs, de quelque sorte qu'ils soient (elle n'a pas accompagné George Nelson).

28. "Accrochée au rideau de ma chambre. Collée sur la vitre" (197); il est à noter qu'Élisabeth d'Aulnières est toujours couchée dans la chambre de Léontine Mélançon, à Québec; même le lecteur en arrive à l'oublier.

29. Ce qui rappelle un peu la campagne de Sorel où habite George Nelson: “ terre vaste, plate, longue. Le comté de Richelieu, à perte de vue. Comme s'il n'y avait plus d'horizon nulle part. Je crains d'avancer en terrain découvert. Jusqu'à cette maison de bois isolée dans les champs" (142).

30. “...emporté dans son traîneau, [iil] a franchi toutes les frontières humaines. II s'enfonce dans une désolation infinie. Comme un navigateur solitaire qui se dirige vers la haute mer»(197). 
Cette tentative de retrouver avec George Neison la plaine de Kamouraska est une nécessité qui semble inhérente au songe: "Fouiller l'horizon le plus loin possible. A la limite extrême de l'attention. Jusqu'à Kamouraska; il le faut » (189). Voir de Sorel jusqu'à Kamouraska: prendre possession de l'espace, d'abord par les yeux. On va retrouver tous les sens qui transportaient Élisabeth d'Aulnières dans les lieux de son passé; cette fois, ils la transportent dans des lieux qu'elle a, en effet, connus, mais non dans le temps où elle les a connus.

S'il y a nécessité d'occuper cet espace, sa volonté y joue aussi un rôle et cette volonté d'être avec George Nelson s'exprime dans un hommage à la "détermination farouche" de cet homme, comme si ce tribut allait fortifier et exaucer ses désirs. De toute façon, la fin de la citation montre que la détermination d'être là et de tuer n'est pas moindre chez la maîtresse que chez l'amant:

Je voudrais respirer l'odeur de la laine grillée. Dans la salle de l'auberge de Saint-Vallier. M'approcher de l'homme penché sur le feu. Le surprendre de dos. Examiner à loisir la nuque de mon amant. J'ai toujours été persuadée que le siège de la volonté et de l'énergie chez l'homme se trouvait logé dans sa nuque. Une telle détermination farouche dans la nuque, à la fois fine et robuste, de George Nelson. Le secret même de sa force, caché là sans doute. Cela m'émerveille et me désespère. Je voudrais posséder mon amour, comme ma propre main. Le suivre dans toutes les démarches de sa vitalité extraordinaire. Que pas une de ses pensées ne m'échappe. Que pas une de ses souffrances ne me soit épargnée. Être deux avec lui. Double et féroce avec lui, lorsqu'il le faudra. Tuer mon mari avec lui (200).

Ce désir de «tuer... avec lui » est déjà une réalité dans l'écriture où les derniers infinitifs, s'ils dépendent syntaxiquement de «je voudrais...", apparaissent comme des affirmations, à force d'être répétés et séparés les uns des autres par une ponctuation très serrée. Ce désir est aussi une réalité dans cette conviction que les lieux vont s'en faire les complices: "Les lieux eux-mêmes [de Sorel à Kamouraska et de Kamouraska à Sorel] me seront largement ouverts, afin que j'entre et sorte, au gré des événements "(184).

Cette étrange assurance de pouvoir habiter des lieux connus, dans un temps où elle n'y était pas, s'appuie sur le pouvoir du songe-souvenir de la conduire dans des lieux qu'elle n'a jamais connus. C'est l'été où elle a rencontré George Nelson; le tintement d'une clochette non seulement provoque la réminiscence d'une situation qu'elle n'a pas vécue, mais la transporte dans le dortoir du collège où Antoine Tassy et George Nelson font leurs études. Elle entend la sonnerie du réveil dans le dortoir même; elle «se souvient» de l'odeur; elle revoit les jeux d'ombre et de lumière créés par la bougie qu'on allume et par les formes vagues qui «sortent du noir. Passent devant la lueur de la bougie. Font des ombres gigantesques et flasques sur le mur. Frissonnent. Replongent dans le noir. Se confondent avec leurs ombres sur le mur»(150); elle voit aussi «une ombre de main [qui] esquisse un signe de croix dans le vide», mais cette "ombre de main pieuse» est engloutie par le mur et cet évanouissement des images 
du songe est suivi de sons: la voix de George, "à peine plus jeune», et une voix empruntée à l'aîné des fils d'Élisabeth et d'Antoine; la voix de cet enfant ramène à la réalité de Sorel et finit le songe, comme il avait commencé, par un son ${ }^{31}$. Cette incursion dans un passé inconnu ne peut, au point de vue des structures de succession, que brouiller les pistes et rendre plus plausible, plus «réelle", l'incursion dans le passé connu de l'enfance et des amours heureuses à Sorel, car la fin de l'épisode dans le «dortoir endormi » nous ramène au présent de Sorel et non à celui de Québec. En même temps, cette fusion des mémoires de George Nelson et d'Élisabeth d'Aulnières, à partir, entre autres, d'éléments sonores, et la "présence" de l'un à la source des souvenirs de l'autre permettent d'envisager la possibilité que le songe rapprochera les corps, dans le temps et dans l'espace, à partir, cette fois encore, de sons et de voix, même si les deux amants ne sont jamais allés ensemble à Kamouraska.

Des voix la tirent de Québec et de Sorel et risquent de la conduire dans une des auberges qui bordent la route de Kamouraska. Elle se rappelle la voix et la déposition, au procès, de l'hôtelier de Saint-Vallier et elle "tente de prendre pied dans l'auberge", où un jeune homme étrange a passé la nuit, le mardi, 29 janvier. La voix lui signale un lieu pour qu'elle l'occupe ${ }^{32}$ et continue à son propre rythme, mais le lieu disparaît de la vue d'Élisabeth d'Aulnières qui ne peut qu'occuper le temps: "Déjà la voix de l'hôtelier enchaîne si rapidement que je suis précipitée dans le temps. À la vitesse même de la parole. Sans pouvoir m'accrocher à aucune image. Ne reconnaître aucun visage"(199). Elle ne pourra pas davantage prendre pied dans cette auberge, le vendredi, 3 février, au retour de George Nelson; elle ne pourra, comme on l'on vu, que manifester son désir de "respirer l'odeur de la laine grillée ", s'approcher, etc.

C'est la lumière qui, inexplicablement encore une fois, ouvre au regard d'Élizabeth d'Aulnières un lieu tout entier, à un moment où elle n'y était pas :

Je m'étonne de pouvoir supporter la neige, en plein soleil. L'éclat aveuglant du ciel bleu. Sainte-Anne-de-la-Pocatière. Le 31 janvier 1839. Ce jour-là entre tous. Comme je vois bien tout. En entier et en détail. Les plus petites mottes de neige projetées par les sabots du cheval. Cette espèce de fumée blanche escortant le traîneau. La grand-route droite, aplanie par le passage des traîneaux. Un instant, une figure d'homme rougie par le froid se tourne vers moi. Est-ce moi qu'il regarde, cet homme (205) ?

Quand la «figure d'homme» la regarde, serait-elle déjà présente physiquement à Sainte-Anne-de-la-Pocatière, le 31 janvier 1839, tout en attendant son amant à Sorel et en revivant cette attente, à Québec, vingt ans après?

31. II y a aussi, juste avant, le son de la glace qu'on casse dans un broc.

32. La façon dont elle décrit cette voix en dit long sur le temps qui est perçu comme un espace en profondeur, un espace où la vie se "déplace": "Quelque chose de vivant bouge, se déploie au fond du silence. Remonte à la surface. Éclate comme des bulles sourdes dans mon oreille. Une voix d'homme, lente..." (199). 
Évidemment, il faut tout mettre sur le compte de l'hallucination. Elle pourrait se donner le même avertissement qu'elle donne à George Nelson, à la fin du paragraphe précédent: “Attention au vertige ${ }^{33}$ !" De même, lorsque le voyageur, lors de la déposition de Louis Clermont, arrive à Saint-Anne et qu'elle croit voir "des taches sombres sur le manteau, en plaques poudrées de neige"(214), en se demandant si ce n'est pas causé par la lueur de la bougie.

$A$ un autre moment, elle tend les bras, dans son lit de la rue du Parloir, à Québec, et touche, à Sorel, les murs de bois de la salle d'attente du docteur, construits en planches de pin qu'elle pourrait compter ${ }^{34}$ - il faut noter que les murs de la chambre de Léontine Mélançon sont recouverts de "papier à fleurs" (40) - : sur le compte de quoi faut-il mettre cette exacerbation du sens du toucher, en rapport avec un lieu qu'elle a bien connu, mais au moment où s'achève justement ce voyage en des événements qu'on a vécus à des milles de distance?

On a déjà vu sa certitude de pouvoir entrer dans les lieux et d'en sortir au gré des événements ${ }^{35}$, événements qu'elle a vécus de très loin, répétonsle; il ne s'agit pas seulement, pour elle, d'en entendre des sons, d'en voir des images ou d'en sentir les odeurs. Le texte revient au moins deux fois sur cette assurance et insiste sur la participation réelle, sinon active, d'Élisabeth d'Aulnières à ces phénomènes: les témoins, selon elle, «projettent de [l']emmener de force jusqu'à Kamouraska" (209) et elle sera tirée de son lit, “mise debout, tout habillée. Sortie de [sa] maison" (207) et «déportée sur Kamouraska” (209); elle ne sait pas si elle sera morte ou vive, mais sait qu'elle n'y échappera pas, tout en devenant «hors de la portée de Jérôme Rolland. Hors d'atteinte de tout ce qui respire encore" (207). En somme, elle sera morte à la vie de l'aujourd'hui, celui de Mme Rolland, et elle sera «placée dans la neige. Le froid. En vigie silencieuse et passive. [Se] relayant... d'auberge en auberge» (207), placée au milieu du temps et de l'oubli d'où elle finira par ramener George Nelson ${ }^{36}$.

Tout cela peut être mis sur le compte de la fiction, de l'image et de l'hallucination, comme lorsqu'elle se dit: "Lancée avec [George Nelson] sur des routes de neige, jusqu'à la fin du monde» (195). On peut y voir aussi une façon de se remémorer les dépositions des témoins, quand elle note: "Avide et folle, j'écume les routes gelées et le temps à jamais écoulé. Pour interroger les aubergistes et les rares voyageurs», bien qu'elle ajoute: "dans l'espoir insensé de retrouver..." (224). Si, dans son esprit, elle semble bien avec lui, sur la route de Kamouraska, lorsqu'on lit: «Depuis hier [bien

33. Au sujet de son vœu d'être un saint, vœu qu'il réaliserait, selon elle, en faisant passer l'absolu qui le ronge "dans le crime et le sang", en lavant ses mains de ce sang et en « revenant avec son beau visage vainqueur de la mort»; cf. p. 129, 177 et 205.

34. "Mes deux bras tendus dans l'ombre. Sous mes doigts surgit un certain mur de bois. Puis un autre mur de bois. Un angle parfaitement droit. Je pourrais compter les planches de pin " (242).

35. Ct. p. 184 et ci-haut, p. 254.

36. Cf. p. 209: «Moi seule pourrais ramener cet homme à la vie. Le tirer hors du temps et de l'oubli. » 
avant l'anse de Kamouraska], tu n'as absorbé aucune nourriture. Je te suis à la trace. Tu entends derrière toi les clochettes de mon attelage. Je suis Mme Rolland " (223), par contre, la technique du songe refait surface quand on lit aussitôt après, dans la même ligne du texte: "Je te hante, comme tu me hantes. Nous délirons tous les deux. La séparation entre nous a déjà eu lieu.»

Cependant, ce délire à deux a-t-il lieu, en même temps, pour Mme Rolland à Québec et pour George Nelson, quelque part aux États-Unis, l'année de la mort de Jérôme Rolland? Rien de moins probable. Il s'agit plutôt du délire de Mme Rolland à Québec et de celui de George Nelson en route pour Kamouraska, en janvier 1839; le délire ou la hantise n'est pas d'imaginer la réunion à travers le temps des deux êtres, mais c'est, pour eux, de croire qu' «écumer le temps à jamais écoulé" et retrouver l'être aimé suffiraient à annuler la séparation qui a déjà eu lieu.

Quoi qu'il en soit, Élisabeth d'Aulnières perçoit comme une réalité le fait d'être dans les auberges, le long de la route. Un témoin, Victoire Dufour, peut jouer d'un tablier qui s'agrandit, grossir, emplir «la chambre [de l'auberge] de sa personne grasse et bleue» et feindre "de s'être levée de bon matin pour aller témoigner chez le juge de paix», et on peut y voir une situation à peu près identique à celle des débuts du songe où des aiguilles de lumière deviennent de petits personnages dans les yeux de $\mathrm{Mme}$ Rolland, avant de prendre taille humaine. Mais la narratrice et le lecteur se trompent, la situation n'est pas la même: «En réalité [Victoire Dufour] s'adresse à moi, Élisabeth d'Aulnières. Cherche à me retenir le plus longtemps possible dans l'auberge de Louis Clermont ${ }^{37}$ "(218). D'ailleurs, elle se disait, un peu avant, "enfermée dans l'auberge de Louis Clermont. Poussée dans l'escalier. Pressée de franchir la porte de la chambre du voyageur. La franchissant, cette porte, à [son] corps défendant » (217).

Que veut dire "être ici " ou "être là", quand ici et là deviennent interchangeables, quand plusieurs lieux lointains s'entremêlent les uns aux autres, quand le présent et le passé se confondent, quand le récit d'un événement vécu loin de soi devient le récit d'un événement qu'on a vécu sur les lieux mêmes? Songe, souvenir tronqué, hallucination, délire, mais aussi perte de la notion courante d'espace au profit de la soumission de l'espace à une face cachée de l'être.

Lorsque le moment du meurtre arrive ${ }^{38}$, elle est prête à attendre "au bord de la route... que l'exécution d'Antoine soit terminée" (223); elle ne veut pas suivre les deux hommes en traîneau, "sur le chemin du roi. En direction de la petite maison de Paincourt ${ }^{39}$ ". Elle sent le traîneau noir qui la "frôle au passage". Elle raconte par la suite comment le meurtre a lieu, ce que dit George Nelson au moment de tuer: “...voilà des nouvelles

37. A Sainte-Anne-de-la-Pocatière.

38. Le paroxysme dans la sensation d'un espace lointain et d'un événement auquel on n'a pas assisté, est évidemment atteint au moment du paroxysme de l'action psychologique: le meurtre.

39. Maison qui servait jadis à ses ébats amoureux avec Antoine. 
de ta femme qui est à Sorel» (234); elle le sait par Nelson qui le lui a raconté à son retour à Sorel.

Le premier temps du meurtre se répète dans son récit et, cette fois, elle entend le bruit de la première détonation: «Dans les sifflements du vent. Je crois que je porte mes deux mains à mes oreilles." Cette incertitude sur le geste qu'elle fait, met en cause sa mémoire au sujet d'un événement dont elle n'a pas été témoin, et accentue d'autant sa présence au moment où elle le vit. Elle continue en décrivant les battements précipités de son cœur, ce qui est sans doute un symptôme de son délire, à Québec, mais elle ajoute: "Nul autre bruit perceptible à cent lieues à la ronde. Trois hommes se rangent pourtant avec moi au bord de la route. Menacés par un train d'enfer [prétendent-ils] qui fonce sur nous au grand galop d'un cheval noir. "Elle devient témoin parmi les témoins, mais un témoin qui refuse de témoigner: "Quant à moi, je suis sourde et aveugle et ne puis vous assurer de rien»: aveugle, pour dire que «ce n'est qu'un homme debout dans son traîneau, conduisant un blessé, en direction de l'anse de Kamouraska», et sourde au point de l'entendre chanter «à tue-tête pour couvrir une plainte sourde, dans le fond du traîneau, sous les robes de fourrure ".

Entend-elle la seconde détonation qui "résonne très loin, dans l'anse»? Ce coup de feu est isolé dans son discours et n'est relié à aucun témoignage, aucun récit, aucune réaction.

Mais elle voit le meurtrier qui «s'acharne, à coups de crosse de pistolet, sur un mort couché, la face dans la neige. Il frappe jusqu'à l'usure de la force surhumaine en lui déchaînée". Elle est tout près du meurtrier, son bras est près de son visage: «Un instant le vainqueur essuie son visage sur ma manche ${ }^{40}$. Lui, il ne la voit pas; il «cherche dans son cœur la femme pour laquelle...»

Serait-ce un effet de la puissance de l'image qui se grave dans la mémoire de multiples façons, que l'on vive un événement intensément ou distraitement, qu'on entende un récit de la bouche de témoins, qu'on le réinvente à sa manière à partir de quelques éléments glanés ici et là, qu'on invente de toutes pièces la réalisation de ses désirs à partir d'expériences passées ou qu'on exacerbe son désir au point de le voir prendre forme, de le sentir et de le toucher dans ses songes ou ses rêveries? Mais la lucidité d'Élisabeth d'Aulnières empêche de la voir uniquement soumise au pouvoir des images. Elle note leurs mouvements, leurs qualités picturales et même leur présence «physique». Les images sont comme des morceaux d'espace qui s'insèrent dans d'autres espaces: "L'image de l'anse qui est au nord-est de Kamouraska, en allant vers Rivière-Ouelle, s'installe dans l'auberge de Louis Clermont» (212). Elles se transmettent à partir de la mémoire d'un témoin ou de George Nelson, en devenant visibles sur leurs yeux, et se propagent comme des ondes, sur le visage d'Élisabeth: "Laissée là 41 toute

40. Déjà, à la page 58 , on avait une substitution de pronoms qui, cette fois, montrait la réalité de Mme Rolland et aussi celle de la communiante qu'elle était: "Dans sa main lourde, dans mon bras pétrifié [...] Une enfant qui est moi me regarde....

41. Dans l'auberge de Louis Clermont, dans la chambre du voyageur. 
seule dans l'obscurité. Percevant les terribles frissons de cet homme. Éprouvant à même mes nerfs tendus l'incomparable insomnie de cet homme. Devinant l'insoutenable journée, récapitulée dans les ténèbres. Les images précises passant devant les prunelles exorbitées du voyageur, couché là sur le lit. Les sentant, ces images, voleter devant ma face, pareilles à des chauves-souris ${ }^{42}$ »(217).

Cette vie propre aux images est un événement du voyage dans le temps entrepris et subi par la narratrice, voyage où les espaces se compénètrent et se fusionnent, par l'intermédiaire de ces images. La sensation d'être présente dans ces espaces aux souvenirs non vécus, l'emporte sur le jeu des images qui en sont la conséquence et non la cause.

Ce n'est pas comme témoin qu'elle est "admise " dans ces lieux, mais comme «voyante et complice» (210): elle participe aux événements et elle voit tout. Si tout n'est qu'image, elle est au centre des images et quand elle voit des images, elle voit ce qui «s'imagine» dans le souvenir des habitants des lieux. Elle qui voulait «fouiller l'horizon le plus loin possible» (189), elle est maintenant devenue centre de l'espace et cercle entourant l'espace: «...placée, immobile et silencieuse, au centre de la maison. Afin que je voie tout et que j'entende tout. Nulle part en particulier et partout à la fois. Dans la salle et dans les chambres " (210).

Cette ubiquité va de pair, on l'a vu, avec une nécessité: «Tolérée dans l'auberge. Pas vraiment acceptée. Mais tolérée seulement. En fait, inévitable. Imposée dans cette auberge de Louis Clermont, à Sainte-Anne-de-laPocatière. Par une implacable volonté. Déposée là comme un paquet. Introduite de force dans l'intimité de cette maison barricadée" (210). Devant une telle suite de termes, où Élisabeth d'Aulnières devient une chose que l'on manipule, on ne sait plus s'il est encore utile de tenter une explication à tous ces phénomènes de déportation et d'ubiquité et s'il ne vaut pas mieux se contenter de les accepter comme un fait inexplicable, «inévitable ".

Ce fait attaque la résistance physique de Mme Rolland et la perception qu'elle a d'elle-même. Elle se dit «réduite à [son] état le plus lamentable» (213). Elle est tantôt prête à se voir presque morte: "Étant le plus près possible qu'il me soit permis de l'être [sans mourir tout à fait] de mon propre néant " $(213)$; tantôt, elle ne voit que la mort pour décrire ce qu'elle ressent: "Me répéter que je suis morte [que rien ne peut plus m'atteindre]. Non point blessée, ni même mourante, mais morte tout à fait» (214). Cette mort et cette presque mort sont le résultat de son invisibilité, de sa perte

42. Si l'on peut rétorquer qu'elle «devine» les images qui passent devant les prunelles de George Nelson, elle ne peut "deviner" qu'elle les "sent" voleter de son visage. Une même forme verbale, le participe présent, est employée pour la narratrice et pour les images ("passant"), ce qui donne aux images un statut d'agent presque équivalent à celui d'Élisabeth d'Aulnières ou, pour le moins, facilite le passage, dans le songe, du virtuel au réel. $\AA$ un autre moment, une image "s'échappe du sommeil lourd de Blanchet, le vagabond" (212); dans la même page, elle dit: "Si je suis brusquement ramenée dans le bas du fleuve, c'est la faute du vagabond, couché là, sur le banc, renâclant son ivresse. Sa pauvre cervelle est visitée par un souvenir précis qui revient sans cesse et hante son sommeil. " 
«de toute forme, de toute épaisseur ou profondeur ${ }^{43}$ » (213); elle se compare à une goutte d'eau 44 ; "cachée dans cette auberge", elle n'a plus de nom, ni de visage, ce qui équivaut pour elle à être «détruite. Niée " (215).

Dans cet état de paralysie physique, et non d'inconscience ${ }^{45}$, elle constate qu'il lui reste au moins l'usage d'un ${ }^{46}$ sens, l'odorat: "L'odorat part en flèche, trouve sa proie. La découvre et la reconnaît. Lui fait fête. Accueille l'odeur de l'assassin. La sueur et l'angoisse, le goût fade du sang. Ton odeur, mon amour, ce relent fauve" (215). L'un de ces sens qui lui ouvrent les lieux du passé et qui lui font vivre des événements qu'elle n'a pas vécus, sauf de loin, témoigne d'une vie propre à ce voyage hors du temps: Élisabeth d'Aulnières vit bien ailleurs, du moins par son odorat. Et par ses yeux: II faut se rappeler aussi qu'elle voulait respirer l'odeur de Nelson ${ }^{47}$, lorsqu'elle «tente de prendre pied dans l'auberge de Saint-Vallier " (199); elle domine l'espace par le songe, au point de réaliser son désir dans une autre auberge, celle de Louis Clermont, à Sainte-Anne. À l'instar d'Élisabeth d'Aulnières qui se demande: "Est-ce possible que je rêve la passion d'un autre, avec cette acuité insoutenable ${ }^{48}$ ? ", on peut se demander s'il est possible qu'elle «rêve" l'accomplissement de ses désirs avec une telle acuité.

Le dernier lien de Mme Rolland, à Québec, avec les événements du meurtre, est un son 49 : le galop d'un cheval. Elle vient de dire à Léontine Mélançon qu'elle a «terriblement besoin de sommeil et de rêve» (219) et elle se retourne contre le mur: «Un galop de cheval se lève comme la poudrerie dans l'anse de Kamouraska, balaye tout de son train d'enfer. Me poursuit! Va me renverser! Me tuer! Je suis hantée. » Elle qui, tout à l'heure, était au centre de l'auberge de Louis Clermont, se dit maintenant l'espace de ses hantises. Tous ces lieux sont en elle, comme elle est dans ces lieux; ces espaces enfermés en elle vont aussi se refermer sur elle.

L'impossibilité de faire quelque mouvement que ce soit vers Nelson, lorsqu'elle revit avec lui son voyage à Kamouraska, en même temps qu'elle marque la limite extrême des pouvoirs du songe, lui fait ressentir l' «inima-

43. Cf. aussi p. 214-215. Si être invisible équivaut à sa mort, être vue était le lot de sa vie: “C'est cela ma vraie vie. Sentir le monde se diviser en deux haies pour me voir passer. La mer Rouge qui se fend en deux pour que l'armée sainte traverse. C'est çà la terre, la vie de la terre, ma vie à moi »(8).

44. "Transparente comme une goutte d'eau" (215); aussi : "Je deviens translucide $"(213)$.

45. "Si je dois souffrir tout ce qui va suivre (et je le dois) ce sera à l'extrême limite de l'attention » (213).

46. Elle ajoute aussitôt le regard: «Un seul sens libéré, agissant. Les quatre autres retenus, entravés. [Sauf le regard, bien entendu.]" (215) ; avant, elle disait: «Et pourtant quelque chose d'irréductible en moi s'élance, hors de moi, lors même que je n'existe plus.»

47. Cf. p. 200 et ci-dessus, p. 254.

48. Cf. p. 199; elle éprouve alors l'engourdissement par le froid qu'aurait subi Nelson, sur la route de Kamouraska.

49. La première fois qu'elle se retrouve dans un lieu qu'elle ne connaît pas et qu'elle "revit " une situation qu'elle n'a pas connue (ou, plus simplement, qu'elle revit les souvenirs d'un autre), c'est aussi par l'intermédiaire d'un son: le tintement de la clochette dans le dortoir du séminaire de Québec (cf. p. 150 et ci-dessus, p. 254-255. 
ginable solitude" (218) de George Nelson et la sienne propre qui restreint l'espace [fût-il celui de la plaine de Kamouraska] à elle-même: "Me voici emmurée dans ma propre solitude. Figée dans ma propre terreur. Incapable d'aucun mouvement, d'aucun geste [...] Je ne puis plus faire un pas vers toi..." (218)

L'anse de Kamouraska, où elle voit avec Blanchet et Élie Michaud «du sang sur la neige, tout le long du chemin de la batture» (226), devient une sorte de sablier où elle a l'impression d'être enfermée ${ }^{50}$, à cause, sans doute, de «cette espèce de songe qui [leur] passe sur la face, avec les flocons" (225); le songe engendre un songe qui l'isole au plein milieu de la neige. Elle est «poussée à la limite extrême du cauchemar " (231), car, autour d'elle, l'espace du songe l'emporte et l'enferme au point où elle n'a plus de refuge, plus de lieu où habiter, n'étant plus en elle-même et étant là où elle ne devrait pas être, là où même sa dignité l'empêcherait de se présenter: "Anachronique et insolite. Tenue de se présenter en personne au manoir de Kamouraska" (230); "Sans aucun refuge à l'intérieur de soi. Chassée hors de soi. Jetée dehors [...] N'ayant jamais été aussi profondément séparée de soi-même ${ }^{51}$ "(231). L'espace devient le songe et détruit comme le songe le faisait. On a ici l'aboutissement des effets créés en elle par les lieux qu'elle retrouve tout au long du songe-souvenir.

L'espace apparaissait réduit et fermé: «Apprendre à vivre en soi ${ }^{52}$. Dans un espace restreint, mais parfaitement habitable. Éviter de regarder à plus de deux pas devant soi. Georges, Aurélie et moi, nous nous exerçons à ramener les quatre coins cardinaux ${ }^{53}$ sur nous. Les réduisant à leur plus simple expression. Moins que les murs d'une chambre. Une sorte de coffret hermétique. Une bouteille fermée. Nous apprenons à respirer le moins profondément possible»(182). En même temps qu'elle trouvait cet espace «parfaitement habitable», parce qu'elle tenait malgré tout à y revivre, elle avait de la difficulté à y respirer: "Je respire un air raréfié, déjà respiré. Je mets mes pas dans mes pas " (100). Elle parlait même de «vide absolu», mais d'un vide absolu qui contient la poussière du passé et un «air gris»: "Une cloche de verre où persiste une sèche poussière, pour m'étouffer. I Dans cet espace réduit, dans cet air gris qui se raréfie... » (58).

Ce vide absolu où, pourtant, elle respirait, indiquait plus la coupure totale d'avec la réalité quotidienne et actuelle, qu'une coupure d'avec la vie. C'était déjà le sablier de la plaine de Kamouraska, sous la neige ${ }^{54}$. Cette coupure du réel était aussi une violence qui lui était faite; avant

50. «On est comme enfermés dans un sablier où tourbillonne la neige douce ( 225$)$.

51. Ailleurs, elle est enveloppée par le temps: "Le temps, le temps, dure, s'étire, m'enveloppe, me traîne avec lui » (247).

52. Le temps passé se refermait sur elle: «Un certain temps de ma vie, réintégré comme une coquille vide. S'est refermé à nouveau sur moi. Un petit claquement sec d'huître. Je m'entraîne à vivre dans cet espace réduit » (100).

53. Cf. «la Chambre fermée», dans Anne Hébert, Poèmes, Paris, Seuil, 1960, p. 40: «Les quatre points cardinaux / Originent au bout de mes doigts..."

54. Elle se sentait comme dans un aquarium, à un autre moment: «Ne pas appeler en vain dans ma cage de verre, ouvrir et refermer la bouche comme les poissons rouges dans leur aquarium»(186). Cf. aussi p. 152-153: "Une eau profonde, infranchissable» entre elle et «cet autre prisonnier de la pluie», George Nelson. 
d'être chassée hors d'elle, séparée d'elle-même (cf. p. 231), elle s'était vue prisonnière de tous ces lieux clos: "Comment faire pour le rejoindre? Je suis encombrée. Surchargée. Ligotée. Prisonnière de la rue Augusta et de la ville de Sorel ${ }^{55}$ "(123). Elle réussissait, comme on sait, à se défaire de ces liens, à retrouver George Nelson et, aussi, la plaine de Kamouraska qui, finalement, se referme sur elle comme une autre prison.

Toutes ces prisons lui enlèvent surtout la sensation ou même la possibilité d'habiter le temps présent: "La même ${ }^{56}$ inflexible convocation, hors du temps [...] Élisabeth d'Aulnières [âgée de toute sa vie, hallucinée, sortie de son temps réel], se fond, se perd... »(230-231).

Ce voyage dans le temps, craint et désiré à la fois, ramène à soimême: «J'apprends l'absence, jour après jour, nuit après nuit. Dans la chambre de la rue Augusta ${ }^{57}$, je vis, à nouveau, comme une prisonnière" (247), ce voyage aboutit dans les ténèbres; Élisabeth d'Aulnières espère encore elle ne sait quoi de ces ténèbres et elle refuse de reprendre pied à Québec, rue du Parloir: "J'empêche la vie et la mort de la rue du Parloir d'arriver jusqu'à moi. Je construis des barrages d'obstination et de mauvaise volonté. Je persiste du côté des ténèbres. Je fouille les ténèbres" (242). Elle retrouve ces ténèbres qui étaient devenues leur lot, à George Nelson et à elle, quand il était revenu de Kamouraska: "Debout, l'un en face de l'autre. Nous nous emplissons de ténèbres " (241).

Des ténèbres qui séparent. Les ténèbres de George Nelson sont d'abord «un désert d'arbres et de neige ${ }^{58}$ » et «l'enlisement calme dans un silence étrange et désolé " (238) qui mènent "de l'autre côté du monde ${ }^{59}$ ". L'autre côté du monde, ce peut être Burlington où il a été emprisonné ${ }^{60}$, mais c'est surtout: «Au cœur même de la malédiction de la terre. Plus qu'un pays étranger. La terre entière étrangère. L'exil parfait. La solitude des fous « (248). Élisabeth d'Aulnières, depuis le début du songe-souvenir, affirme qu'il n'y a rien de commun entre elle et cet «autre côté du monde " et que tout l'en sépare: «Une sorte de désert se creusant entre nous. Le silence. Le vide. [...] De nouveau un mur entre nous, bien lisse et dur ${ }^{61}$ " (123). Plus loin, quand elle se rappelle avoir assisté, assise à une fenêtre, au départ de Nelson pour Kamouraska, elle reprend cette image du mur:

55. Cf. tout le premier paragraphe p. 106 et aussi p. 96: "Je n'ose avancer, franchir cette clôture invisible. Dressée autour de moi, "

56. Comme il a été dit au début, ce voyage dans le temps n'est pas le premier et sans doute n'est pas le dernier.

57. À Sorel; on revient aux souvenirs vécus.

58. «James Wood et Robert Dunham ne sont pas loin de croire aux vertus surnaturelles du grand cheval noir emportant son démon de maître à l'intérieur des terres. Sur des chemins peu sûrs. Dans un désert d'arbres et de neige» (238).

59. Cf. p. $189,218,248$ et aussi Anne Hébert, les Chambres de bois, p. 110: «Catherine se demandait quelle puissance souveraine venait ainsi saisir la jeune femme au milieu de si pauvres soirées et, doucement, sous les yeux de Catherine et Michel, la faisait passer de l'autre côté du monde. "

60. "Burlington, Burlington, mon amour m'appelle de l'autre côté de la frontière, de l'autre cốté du monde..." (218).

61. A ce moment-là, elle regrette de ne pouvoir rejoindre George Nelson: elle reculait l'échéance du retour au meurtre, en voulant d'abord revivre ses amours. 
«Désormais, entre nous, il y aura cet écran de verre et de gel. Ton image déformée par le givre et la mort passera de l'autre côté du monde» (189).

Mais elle sait qu'en lui faisant traverser la frontière du crime et de la mort, elle le pousse, elle-même, de l'autre côté du monde et qu'elle peut seulement feindre «d'appartenir au monde des vivants» (248); sa "plus profonde allégeance " à elle aussi, est d'être "étrangère et possédée " et le songe en apporte la preuve. Par Antoine Tassy, elle appartient au monde, à l'espace des morts; elle l'entend qui tente de hurler «du fin fond du bout du monde et de la mort» (111) et «qui geint doucement les nuits de tempête» (80); elle l'imagine (ou le voit?) qui «suit des couloirs sous terre. Des chemins noirs là où passent les eaux souterraines " $(80)$; elle le sent comme un géant qui se brise contre son épaule, «Vole en éclats. Envahit tout [son] être. Des milliers d'épines dans [sa] chair» et la suite montre jusqu'à quel point le mort Antoine Tassy et elle ne font qu'un:

Je suis hantée, jusqu'à la racine de mes cheveux, la pointe de mes ongles. Antoine multiplié à l'infini, comme écrasé au pilon, réduit en fines particules. Chaque grain infime conservant le poids entier du crime et de la mort. Son rang répandu. Sa tête fracassée. Son cœur arrêté. [...] Son sang, sa tête, son cœur. Cela recommence. Une ronde dans mes os, une multitude d'Antoines assassinés circule dans mes os (92).

Cette hallucination où le mort est mêlé à son sang, le songe peut en indiquer le pendant dans la vie réelle de Mme Rolland: le désert du monde. Quand elle se rappelle ses retours, au lever du jour, à la maison de la rue Augusta, après avoir quitté George Nelson, elle songe au «désert du monde" (160); par la suite, revenant à sa situation d'épouse de monsieur Rolland et à l'absence de son amant, elle ajoute: "Le pire qui pourrait encore m'arriver, c'est d'être condamnée au désert du monde " (160). Cette crainte de vivre pour toujours dans un monde vide, à cause de l'absence de l'amour ${ }^{62}$, le songe la confirme, dans un dernier temps, lorsque, la main dans celle de son mari mourant, elle rêve à une femme, enterrée vivante depuis longtemps "dans un champ aride sous les pierres ${ }^{63}$ », qu'on déterre et lâche dans la ville avec une faim de vivre qui "doit être si féroce et entière, accumulée sous la terre depuis des siècles!"; de toute évidence, cette femme mourra de faim et de solitude, parce qu' «elle ne trouve que des portes fermées et le désert de terre battue dont sont faites les rues" (250). Ce désert de terre battue est le dernier espace qu'on mentionne dans le roman Kamouraska. L'espace physique du dernier cauchemar répond à l'espace «moral » d'Élisabeth d'Aulnières qui foule aux pieds la défroque de Mme Rolland, pour revivre ses amours, et dit: «J'habite la fièvre et la démence, comme mon pays natal " (115).

62. Cf. p. 9-11.

63. Le poème "Retourne sur tes pas" dans Poèmes, (Paris, Seuil, 1960, p. 45-46) pourrait évoquer cette vie sous les pierres et se prêter à beaucoup de comparaisons avec Kamouraska. 
Le délire, dans Kamouraska, donne naissance à l'espace, pour ensuite y habiter. Élisabeth d'Aulnières se refuse, d'abord, aux pouvoirs de ce délire et prétend les soumettre aux lois du songe ou même de la mémoire; elle finit par s'y abandonner, pour occuper tous les espaces qui la hantent.

Ce délire n'agit pas au niveau de sa raison, mais de ses sensations. Les sensations du présent s'enchaînent aux sensations du passé et animent de nombreux espaces enfouis dans la mémoire; ainsi la sensation de la lumière devient la vision d'un lieu et ce lieu même. La vie propre de ces espaces, les sensations qui en résultent chez la narratrice, comme celles qui les ont provoquées, tout cela crée un appel de vie - «ll s'agit de la possession de ma vie réelle» - qui conduit à la recherche, par Élisabeth d'Aulnières, des sensations les plus intenses de sa vie de jeune femme: cet amour éperdu pour le docteur George Nelson.

Atteindre ce but, c'est aussi déboucher sur l'absence de George Nelson; cette disparition provoque, en retour, celle des espaces qui avaient justement provoqué la présence de l'amant. C'est à ce moment que la sensibilité exacerbée d'Élisabeth d'Aulnières doit se créer un autre espace, qui lui soit bien propre, un espace extraordinaire, un «ailleurs » qui soit un «vide absolu", pour qu'elle puisse, par ce néant, retrouver George Nelson qui, pour elle, est passé de l'autre côté du monde, dans les ténèbres.

Elle disparaît, pour ainsi dire, de sa chambre de Québec et des lieux de son enfance, à Sorel, pour que surgisse autour d'elle la plaine de Kamouraska, durant ces jours où elle n'y était pas, et qu'elle l'occupe avec celui qui, dans le passé, s'y trouvait seul avec sa victime, Antoine Tassy.

C'est à ce moment, surtout, que la perception sensorielle extrêmement aiguë qu'elle a de ses souvenirs - son délire? - lui permet de rassembler dans le temps et dans l'espace, des sensations qu'à une époque elle a éprouvées auprès d'un homme, à une autre, au milieu d'un paysage de neige et de glace, à une troisième, au récit d'un événement ou à l'idée qu'elle s'était faite de cet événement, et alors, d'éprouver ces sensations, éparses dans sa vie, comme un seul et même épisode de sa "vraie vie", vécu avec une intensité presque insoutenable et ne pouvant se comparer à aucun autre épisode de sa vie «normale». Elle prend ainsi possession de l'espace; elle se l'approprie. S'il s'agit d'un délire, il faut parler de délire raisonné, prémédité.

Si l'espace se soumet et s'ouvre, pour qu'elle y entre et en sorte, "au gré des événements», il se referme finalement sur elle et la rejette, comme la terre rejette cette femme enterrée vivante. Ni le songe, ni l'espace ne sont encore assez vastes pour contenir la peur de Mme Rolland qui doit demander de l'aide à un mourant: «Si tu savais, Jérôme, comme j'ai peur» (250). 